\title{
CONFORMAL MAPS, MONODROMY TRANSFORMATIONS, AND NON-REVERSIBLE HAMILTONIAN SYSTEMS
}

\author{
XiAnghong Gong
}

\section{Introduction}

In this note we shall consider real analytic Hamiltonians of the form

$$
H(x, y)=\sum_{j=1}^{n} \alpha_{j}\left(x_{j}^{2}+y_{j}^{2}\right)+O(3)
$$

where $(x, y)$ are the symplectic coordinates of $\mathbf{R}^{2 n}$, associated to the symplectic 2 -form $\sum d x_{j} \wedge d y_{j}$. The Hamiltonian vector field of $H$ is

$$
X_{H}=\sum-H_{y_{j}} \frac{\partial}{\partial x_{j}}+H_{x_{j}} \frac{\partial}{\partial y_{j}} .
$$

According to Arnol'd and Sevryuk, a Hamiltonian vector field $X_{H}$ is said to be weakly reversible if $\varphi_{*} X_{H}=-X_{H}$ for some germ $\varphi$ of real analytic transformation with $\varphi(0)=0$, while $X_{H}$ is reversible if additionally $\varphi$ is an involution, i.e., $\varphi^{2}=\mathrm{Id}$. One also says that $\alpha_{1}, \ldots, \alpha_{n}$ are non-resonant, if

$$
k \cdot \alpha \equiv k_{1} \alpha_{1}+\cdots+k_{n} \alpha_{n} \neq 0
$$

for all integers $k_{j}$ with $k=\left(k_{1}, \ldots, k_{n}\right) \neq 0$.

The main purpose of this note is to show the existence of non-reversible real analytic Hamiltonian systems of non-resonant eigenvalues. We shall prove

Theorem 1. For $n \geq 2$ there exist non weakly reversible Hamiltonian vector fields $X_{H}$ of the form (1) for which $\alpha_{1} \cdot \alpha_{2}<0$, and $\alpha_{1}, \ldots, \alpha_{n}$ are non-resonant.

We should mention that any real analytic Hamiltonian on $\mathbf{R}^{2}$ can be put into the Birkhoff normal form, if it starts with a non-degenerate quadratic form; in particular, its corresponding Hamiltonian system is reversible. Also, all Hamiltonian systems (2) are reversible by some formal involution when their eigenvalues satisfy the above non-resonance condition. Arnol'd and Sevryuk [1] gave a Hamiltonian function on $\mathbf{R}^{2}$ with vanishing quadratic form, of which the corresponding Hamiltonian vector field is not reversible by any linear involution. See

Received April 18, 2000.

2000 Mathematics Subject Classification. Primary 37J99, 32S40.

Key words and phrases. Non-reversible Hamiltonian system, conformal map, monodromy transformation.

Supported in part by NSF grants DMS-9704835 and DMS-0096047. 
recent surveys of Roberts and Quispel [7] and Lamb and Roberts [4] on reversible dynamical systems.

Theorem 1 is analogous to the existence of non-reversible area-preserving maps [3], where the hyperbolic orbits of the complexified maps provide an obstruction to the reversibility of the real maps. We shall see that the obstructions to the reversibility of real Hamiltonian systems lie in the complexification of $\mathbf{R}^{2 n}$ also. To seek non-reversible Hamiltonian systems, we shall restrict ourselves to those systems, of which the complexifications admit subsystems defined on some invariant complex submanifolds passing the origin. It turns out that the reversibility of the real Hamiltonian systems implies, in a certain sense, that of the monodromy transformations of those subsystems. The proof of the theorem uses a result of Pérez Marco and Yoccoz [6], which says that any conformal map can be prescribed as a monodromy transformation of holomorphic vector fields in $\mathbf{C}^{2}$. We shall also need the non-reversibility of conformal maps [3].

\section{Reversibility of subsystems}

It is convenient to introduce complex coordinates $z_{j}=x_{j}+i y_{j}$ on $\mathbf{R}^{2 n}$ that are compatible to the symplectic structure $\sum d x_{j} \wedge d y_{j}$. Thus a real analytic Hamiltonian on $\mathbf{R}^{2 n}$ becomes a holomorphic Hamiltonian on $\left(\mathbf{C}^{2 n}, \frac{i}{2} \sum d z_{j} \wedge\right.$ $\left.d w_{j}\right)$. More precisely, rearrange $H(x, y)$ as a power series in $z, \bar{z}$, and denote it by $H^{c}(z, \bar{z})$. Introduce the notation

$$
\bar{f}(Z)=\overline{f(\bar{Z})}
$$

for a multivariable function $f$. Then $H^{c}$ satisfies the reality condition

$$
\bar{H}^{c}(z, w)=H^{c}(w, z) \text {. }
$$

Now, the Hamiltonian vector field (2) becomes

$$
X_{H^{c}}=2 i \sum H_{w_{j}}^{c}(z, w) \frac{\partial}{\partial z_{j}}-H_{z_{j}}^{c}(z, w) \frac{\partial}{\partial w_{j}}, \quad(z, w) \in \mathbf{C}^{2 n} .
$$

Notice that $X_{H^{c}}$ is the unique holomorphic vector field on $\mathbf{C}^{2 n}$ satisfying $\Re\left\{X_{H^{c}}\right\}=X_{H}$ on $\mathbf{R}^{2 n}: w=\bar{z}$. Note that a real analytic transformation $\varphi$ of $\mathbf{R}^{2 n}$ has a unique complexification $\varphi^{c}$, a holomorphic transformation of $\mathbf{C}^{2 n}$, such that $\left.\varphi^{c}\right|_{\mathbf{R}^{2 n}}=\varphi$. Also, $\varphi_{*} X_{H}=\varphi_{*} \Re\left\{X_{H^{c}}\right\}=\Re\left\{\varphi_{*}^{c} X_{H^{c}}\right\}$. This implies that if $X_{H}$ is reversible by a real analytic transformation $\varphi$, then $X_{H^{c}}$ is reversible by $\varphi^{c}$. From now on, we substitute $H, X_{H}, \varphi$ for $H^{c}, X_{H^{c}}, \varphi^{c}$, respectively.

We shall consider holomorphic Hamiltonians of the form

$$
H(z, w)=\sum \alpha_{j} z_{j} w_{j}\left(1+A_{j}(z)+\bar{A}_{j}(w)\right), \quad A_{j}(0)=0,
$$

where $\alpha_{j}$ are non-resonant. Note that $w=0$ is invariant under the flow of $X_{H}$, and that $X_{H}$, when restricted to $w=0$, becomes the holomorphic vector field

$$
v_{a}=\sum a_{j}(z) \frac{\partial}{\partial z_{j}}, \quad a_{j}=2 i \alpha_{j} z_{j}\left(1+A_{j}(z)\right),
$$

while $v_{\bar{a}}$ is the restriction of $X_{H}$ to $z=0$. 
Lemma 2. Let $X_{H}$ be the Hamiltonian vector field (4) with $\alpha_{1}, \ldots, \alpha_{n}$ being non-resonant, and let $v_{a}$ be the corresponding holomorphic vector field (5). Let $\varphi$ be a holomorphic transformation satisfying $\varphi_{*} X_{H}=-X_{H}$. Then $\varphi$ sends $\left(z_{1}, \ldots, z_{m}\right)$-subspace into $\left(w_{1}, \ldots, w_{m}\right)$-subspace and preserves $\left(z_{1}, \ldots, z_{m}, w_{1}, \ldots, w_{m}\right)$-subspace, while $\phi_{*} v_{a}=-v_{\bar{a}}$ with $\phi$ being the last $n$ components of $z \rightarrow \varphi(z, 0)$.

Proof. From the eigenvalues of $X_{H}$ and $-X_{H}$, one sees that the linear part of $\varphi$ sends $\left(z_{1}, \ldots, z_{m}\right)$-subspace onto $\left(w_{1}, \ldots, w_{m}\right)$-subspace. Put $\left(z_{1}, \ldots, z_{m}\right)=z^{\prime}$ and $\left(z^{\prime}, z^{\prime \prime}\right)=z$. Thus $M=\varphi\left(\left\{z^{\prime \prime}=0=w\right\}\right)$ is defined by

$$
z_{j}=f_{j}\left(w^{\prime}\right), \quad 1 \leq j \leq n ; \quad w_{j}=g_{j}\left(w^{\prime}\right), \quad j>m
$$

with $f_{j}\left(w^{\prime}\right)=O\left(\left|w^{\prime}\right|^{2}\right)=g_{j}\left(w^{\prime}\right)$. Since $-X_{H}\left(z_{j}-f_{j}\left(w^{\prime}\right)\right)$ and $-X_{H}\left(w_{j}-g_{j}\left(w^{\prime}\right)\right)$ vanish on $M$, then

$$
\begin{aligned}
& X_{H}\left(z_{j}-f_{j}\left(w^{\prime}\right)\right)=\sum_{k=1}^{n} u_{j k}\left(z_{k}-f_{k}\left(w^{\prime}\right)\right)+\sum_{k>m} v_{j k}\left(w_{k}-g_{k}\left(w^{\prime}\right)\right), \\
& X_{H}\left(w_{j}-g_{j}\left(w^{\prime}\right)\right)=\sum_{k=1}^{n} \tilde{u}_{j k}\left(z_{k}-f_{k}\left(w^{\prime}\right)\right)+\sum_{k>m} \tilde{v}_{j k}\left(w_{k}-g_{k}\left(w^{\prime}\right)\right),
\end{aligned}
$$

in which $u_{j k}, \tilde{u}_{j k}, v_{j k}, \tilde{v}_{j k}$ are holomorphic functions in $z, w$. Comparing the terms that are linear in $z, w$ yields all $u_{j k}(0)=\tilde{u}_{j k}(0)=v_{j k}(0)=\tilde{v}_{j k}(0)=0$, except that

$$
u_{j j}(0)=2 i \alpha_{j}, \quad 1 \leq j \leq n ; \quad \tilde{v}_{j j}(0)=-2 i \alpha_{j}, \quad j>m .
$$

Assume for the sake of contradiction that $f, g$ have a finite vanishing order $s \geq 2$. Setting $w^{\prime \prime}=0=z$ in (6) and comparing terms of order $s$ yields

$$
\left(K \cdot \alpha^{\prime}\right) f_{j K}=-\alpha_{j} f_{j K}, \quad 1 \leq j \leq n ; \quad\left(K \cdot \alpha^{\prime}\right) g_{j K}=\alpha_{j} g_{j K}, \quad j>m
$$

for $K=\left(K_{1}, \ldots, K_{m}\right), \alpha^{\prime}=\left(\alpha_{1}, \ldots, \alpha_{m}\right)$ and $|K|=s$. By the non-resonance condition (3), $f_{j K}$ and $g_{j K}$ vanish. The contradiction shows that $M$ is $\{z=0=$ $\left.w^{\prime \prime}\right\}$. By a similar argument, one can show that $\varphi$ preserves $z_{j}=w_{j}=0$ for $j>m$.

We now know that

$$
\varphi(z, 0)=(0, \phi(z))
$$

where $\phi$ is a holomorphic transformation of $\mathbf{C}^{n}$. When restricted to $z=0$, the identity $\varphi_{*} X_{H}=-X_{H}$ becomes $-v_{\bar{a}}=\left.\left(\varphi_{*} X_{H}\right)\right|_{z=0}=\left.\varphi_{*}\right|_{w=0}\left(\left.X_{H}\right|_{w=0}\right)=\phi_{*} v_{a}$. The proof of the lemma is complete.

\section{Non-reversibility of monodromy transformations}

We shall consider the holomorphic foliations on a punctured neighborhood of the origin in $\mathbf{C}^{n}$, defined by vector fields (5). Two such foliations, defined by $v_{a}$ and $v_{b}$, are said to be equivalent near the origin if there is a holomorphic transformation $\varphi$ sending leave of one foliation into leave of another, i.e, $\varphi_{*} v_{a}=$ $u v_{b}$ for some holomorphic function $u$. Assume that $\alpha_{j}$ are non-resonant. Then 
an argument similar to the proof of Lemma 3 shows that a germ of complex submanifold at origin, which is invariant under the flow, is the linear span of some coordinate axes.

Let us recall the monodromy transformations of $v_{a}$. Let $\Sigma$ be a separatrix (an invariant holomorphic curve of $v_{a}$, passing through the origin). The monodromy transformations associated to $\Sigma$ are defined as follows. Let $\gamma:[0,1] \rightarrow \Sigma^{*}=\Sigma \backslash$ $\{0\}$ be a real analytic curve with $\gamma(0)=\gamma(1)$. Let $C$ be a complex hypersurface transverse to $\Sigma$ at $\gamma(0)$. Then

$$
\dot{\gamma}(t)=u(t) v_{a}(\gamma(t))
$$

Let $z=Z(t, z)$, with the initial value $Z(0, z)=z$, be the solution to the nonautonomous system

$$
\dot{z}=u(t) v_{a}(z) \text {. }
$$

Then $Z(t, p)$ is holomorphic in a neighborhood of the set $[0,1] \times\{\gamma(0)\}$ in $\mathbf{C} \times C$. The holomorphic curve $Z(\cdot, p)$ intersects $C$ transversely at a point $p^{\prime}=Z(t, p)$ for some $t \in \mathbf{C}$ close to 1 . Thus, $p^{\prime}=h(p)$ defines a holomorphic transformation of $C$, fixing $\gamma(0)$. Let $\gamma_{0}, \gamma_{1}$ be two immersed real analytic curves connected by a homotopy $\gamma_{s}$ of real analytic curves in $\Sigma^{*}$, and let $C_{j}$ be a complex hypersurface transverse to $C_{j}$ at $\gamma_{j}(0)$. Then the monodromy transformations $h_{j}$, associated to $C_{j}, \gamma_{j}$, satisfy $h_{1}=f \circ h_{0} \circ f^{-1}$, where $f: C_{0} \rightarrow C_{1}$ is some holomorphic transformation sending $\gamma_{0}(0)$ to $\gamma_{1}(0)$. Thus, one can define the monodromy transformation of the foliation associated to a closed continuous curve $\gamma$ in $\Sigma^{*}$, while the conjugate class of the monodromy transformations depends only on the homotopy class of $\gamma$ in $\Sigma^{*}$. Notice that two foliations on $\mathbf{C}^{2}$, defined by vector fields of the form (5), are holomorphically equivalent, if and only if their monodromy transformations are conjugate ([2], [5]).

The realization theorem of Pérez Marco and Yoccoz says precisely that the conjugate class of any conformal map $\xi \rightarrow e^{2 \pi \beta i} \xi+O(2)$ can be prescribed as a monodromy transformation of some vector field (5) in $\mathbf{C}^{2}$ with $\beta=-\alpha_{2} / \alpha_{1}>0$, where the monodromy transformation is associated to the $z_{1}$-axis and closed curve $t \rightarrow\left(e^{-2 \pi i t}, 0\right)(0 \leq t \leq 1)$; see also a previous result of Martinet and Ramis [5], when $\alpha$ is rational. Thus, we define $h_{a}$ to be such a monodromy transformation of $v_{a}$ given by (5) with $n=2$.

Lemma 3. The monodromy transformation $h_{\bar{a}}$ is conjugate to $\bar{h}_{a}^{-1}$.

Proof. For $z_{2} \in \mathbf{C}$, we have $\left(1, h_{a}\left(z_{2}\right)\right)=Z\left(1,1, z_{2}\right)$, where $Z(t, z)$ is the flow defined by

$$
\begin{aligned}
& \frac{\partial}{\partial t} Z_{1}(t, z)=-2 \pi i Z_{1}(t, z) \\
& \frac{\partial}{\partial t} Z_{2}(t, z)=2 \pi i \beta Z_{2}(t, z) \frac{1+A_{2}(Z(t, z))}{1+A_{1}(Z(t, z))}
\end{aligned}
$$


for $\beta=-\alpha_{j} / \alpha_{1}$. Conjugating the equations and setting $s=1-t$ yields

$$
\begin{aligned}
\frac{\partial}{\partial s}\left\{\bar{Z}_{1}(1-s, z)\right\} & =-2 \pi i \bar{Z}_{1}(1-s, z) \\
\frac{\partial}{\partial s}\left\{\bar{Z}_{2}(1-s, z)\right\} & =2 \pi i \beta \bar{Z}_{2}(1-s, z) \frac{1+\bar{A}_{2}(\bar{Z}(1-s, z))}{1+\bar{A}_{1}(\bar{Z}(1-s, z))}
\end{aligned}
$$

for $\bar{Z}(t, z) \equiv \overline{Z(\bar{t}, \bar{z})}$. Hence, $h_{\bar{a}}$ sends $\bar{Z}_{2}\left(1,1, z_{2}\right)$ to $\bar{Z}_{2}\left(0,1, z_{2}\right)=z_{2}$, i.e., $h_{\bar{a}}^{-1}\left(z_{2}\right)=\bar{Z}_{2}\left(1,1, z_{2}\right)=\bar{h}_{a}\left(z_{2}\right)$. The proof of the theorem is complete.

Proof of Theorem 1. We want to find a real analytic function $H$ of the form (4) such that the corresponding holomorphic Hamiltonian vector field $X_{H}$, given by (5), is not weakly reversible. In particular, the real Hamiltonian vector field $X_{H}$ is not reversible by any real analytic transformation.

We first consider the case $n=2$. By a theorem in [3], there exists a conformal map $F: \xi \rightarrow e^{2 \pi i \beta} \xi+O(2)$ with $\beta>0$ being irrational, such that $F$ and $\bar{F}^{-1}$ are not equivalent by any holomorphic transformation. By the realization theorem, $F$ is the monodromy transformation of some vector field $v_{a}$. Let $H$ be the corresponding Hamiltonian of the form (4) with $\alpha_{1}=-\pi$ and $\alpha_{2}=\pi \beta$. Assume for the sake of contradiction that $X_{H}$ is weakly reversible, that is that $\varphi_{*} X_{H}=-X_{H}$ for some holomorphic transformation $\varphi$. Put $\varphi(z, 0)=(0, \phi(z))$. Lemma 3 implies that the two foliations defined by $v_{a}, v_{\bar{a}}$ are equivalent through $\phi$, and that $\phi$ preserves the coordinate axes. Obviously, the holomorphic map $\phi$ preserves the homotopy class of $t \rightarrow\left(e^{-2 \pi i t}, 0\right)(0 \leq t \leq 1)$ in the punctured $z_{1}$-axis. Therefore, Lemma 2 and Lemma 3 imply that the monodromy transformation $h_{a}$ is conjugate to $\bar{h}_{a}^{-1}$, which contradicts that $F$ and $\bar{F}^{-1}$ are not conjugate.

For $n>2$, let $H$ be the above real Hamiltonian on $\mathbf{R}^{4}$ such that the holomorphic Hamiltonian vector field $X_{H}$ is not weakly reversible by any holomorphic transformation. Let $G$ be a real analytic Hamiltonian with $\alpha_{1}, \ldots, \alpha_{n}$ non-resonant, of which the restriction to the $\left(x_{1}, x_{2}, y_{1}, y_{2}\right)$-subspace is $H$. If the holomorphic Hamiltonian vector field $X_{G}$ is reversible by a holomorphic transformation $\varphi$, then Lemma 3 implies that $\varphi$ preserves the $\left(z_{1}, z_{2}, w_{1}, w_{2}\right)$ subspace. Hence the complexification of $X_{H}$ is also weakly reversible, which is a contradiction. The proof of the theorem is complete.

Addendum. After the manuscript was submitted, R. Peréz-Marco brought the author the observation that if $F(z)=\lambda z+O(2)(\lambda \neq 0)$ is a polynomial that is not linearizable near the origin, then $F$ and $\bar{F}^{-1}$ are not equivalent by convergent transformations. Peréz-Marco's observation is based on the theory of Fatou and Julia; namely, such an $F$ has repellors accumulating at the origin, while it has only finitely many attractors on the complex plane, from which one readily sees that $F$ and $\bar{F}^{-1}$ are not equivalent by convergent transformations (see Proposition 5.1 in [3]). A theorem of Yoccoz says that $F(z)=\lambda z+z^{2}(|\lambda|=1)$ is not linearizable, if $\lambda$ does not satisfy the Bruno condition. Thus, the construction 
in this paper also shows the existence of non weakly reversible Hamiltonian systems of the form (1), if $\alpha_{2} / \alpha_{1}<0$ and $e^{i \alpha_{2} / \alpha_{1}}$ does not satisfies the Bruno condition.

The author is grateful to R. Peréz-Marco for the above observation.

\section{References}

[1] V. I. Arnol'd and M. B. Sevryuk, Oscillations and bifurcations in reversible systems, in "Nonlinear phenomena in plasma physics and hydrodynamics", "Mir", Moscow, 1986, 3164.

[2] P. M. Elizarov and Yu. S. Il'yashenko, Remarks on orbital analytic classification of germs of vector fields, (Russian) Mat. Sb. (N.S.) 121(163) (1983), no. 1, 111-126.

[3] X. Gong, Conformal maps and non-reversibility of elliptic area-preserving maps, to appear in Math. Z.

[4] J. S. W. Lamb and J. A. G. Roberts, Time-reversal symmetry in dynamical systems: $a$ survey, Phys. D 112(1998), 1-39.

[5] J. Martinet and J.-P. Ramis, Classification analytique des équations différentielles non linéaires résonnantes du premier ordre, Ann. Sci. École Norm. Sup. 16(1983), 571-621 (1984).

[6] R. Pérez Marco and J.-C. Yoccoz, Germes de feuilletages holomorphes à holonomie prescrite, Complex analytic methods in dynamical systems (Rio de Janeiro, 1992). Astérisque No. 222(1994), 345-371.

[7] J. A. G. Roberts and G. R. W. Quispel, Chaos and time-reversal symmetry. Order and chaos in reversible dynamical systems, Phys. Rep. 216(1992), 63-177.

Department of Mathematics, University of Wisconsin-Madison, Madison, WI 53706-1388.

E-mail address: gong@math.wisc.edu 